\title{
Optimal power flow computations with constraints limiting the number of control actions
}

\author{
Florin Capitanescu, William Rosehart, Senior Member, IEEE, and Louis Wehenkel, Member, IEEE
}

\begin{abstract}
This paper focuses on optimal power flow (OPF) computations in which no more than a pre-specified number of controls are allowed to move. The benchmark formulation of this OPF problem constitutes a mixed integer nonlinear programming (MINLP) problem. To avoid the prohibitive computational time required by classical MINLP approaches to provide a (potentially sub-optimal) solution, we propose instead two alternative approaches. The first one consists in reformulating the MINLP problem as a mathematical program with equilibrium constraints (MPEC). The second approach includes in the classical OPF problem a nonlinear constraint which approximates the integral constraint limiting the number of control variables movement. Both approaches are solved by an interior point algorithm (IPA), slightly adapted to the particular characteristics of each approach. We provide numerical results with the proposed approaches on two test systems and for two practical problems: minimum cost to remove thermal congestion, and minimum cost of load curtailment to restore a feasible equilibrium point.
\end{abstract}

Index Terms - mathematical programming with equilibrium constraints, nonlinear programming, optimal power flow

\section{INTRODUCTION}

$\mathbf{S}^{\mathrm{n}}$ INCE most real-time OPF applications run in open loop, the system operator (SO) looks for a practical number of control actions to take in a given time period in order to remove violated operating limits and/or to improve a predefined operation objective [1]-[5]. A very important consideration for the $\mathrm{SO}$ is thus the trade-off between the objective function and the number of control actions used in the optimization. There is no straightforward way to formulate these major concerns in a conventional OPF. Indeed, most conventional OPF computations use the whole set of control means to solve the problem and very often (almost) all of them have moved at the optimal solution. The difficulty of limiting the number of controls moved is due to the fact that: (i) almost every control variable participates in a non separable way to both improving the objective and satisfying the constraints, and (ii) control actions are not easy to rank and the effectiveness of an action is not necessarily related to its magnitude [3].

The OPF problem with limited number of controls allowed to move, which we call hereafter OPFLC, can be formulated as a MINLP problem. To avoid the prohibitive computational time of classical MINLP approaches (e.g., generalized Benders decomposition, branch and bound, etc.) and since the aim is to quickly obtain a reasonable solution, faster heuristic

F. Capitanescu and L. Wehenkel are with the Department of Electrical Engineering and Computer Science, University of Liège, B4000 Liège, Belgium (e-mail: capitane@montefiore.ulg.ac.be; 1.wehenkel@ulg.ac.be).W. Rosehart is with the Department of Electrical Engineering, University of Calgary, Canada (e-mail: rosehart@ucalgary.ca). techniques should be utilized. The problem is likely to be very complex, in particular because for a particular limit on the number of allowed control movements there may exist several combinations of controls ensuring feasibility and satisfying first order optimality conditions.

The simplest and widely used approach to the OPFLC consists in specifying beforehand the controls participating in optimization [1]-[4]. The main drawback of this approach is that it requires very good knowledge about the power system (e.g., based on SO experience) especially in the context of ever changing system states (e.g., generation, load, topology, etc.). In addition, it may happen that an inappropriate choice of controls yields an infeasible OPF problem and hence to the divergence of computations. This requires repeatedly altering the initial set of controls allowed to move and re-running the OPF until convergence. An alternative technique consists of first solving the conventional OPF, then selecting from the OPF output a desired number of controls (e.g., based on some sensitivity information), and performing again the OPF computation with these controls only. This procedure needs also to be repeated until convergence. Note also that the higher the number of control variables moved at the conventional OPF optimum, the more delicate the choice of a significantly smaller subset of control variables.

Only a reduced number of approaches devoted to limiting the number of control actions in an OPF have been proposed in the literature (e.g., [6], [7]). These particular approaches rely on sensitivities of the objective and constraints satisfaction to control movements [6], and on approximating the integral constraint of maximal number of controls allowed to move by one nonlinear constraint, respectively [7].

Given the aim of the OPFLC problem, it may be suitable to formulate its MINLP approach as a MPEC problem [8]. Although the latter approach has been seen for a long time as a very though problem, recent advances in the field of nonlinear programming (NLP) make possible to solve MPEC problems with classical NLP solvers, due to the level of robustness and maturity reached by the latter (e.g., sequential quadratic programming (SQP), interior point method (IPM), active set identification, etc.) [9]-[16]. The main advantage of the MPEC approach, formulated in turn as a NLP problem, is that its time solution is generally polynomial, while the worst case computational cost of MINLP strategies is exponential. This indicates that MPEC problems may scale better than equivalent MINLP formulations on large problems. Obviously, the drawback of the MPEC formulation is that the problem is highly non-convex, due to the large set of discrete equilibria (stemming from strict complementarity of solutions) and hence 
only local solutions can be guaranteed.

Some problems in the area of electric power systems have been formulated as MPEC, most of them being related to gaming within electricity markets [17]-[20], while the application of MPEC in the context of OPF has received much less attention so far [21], [22]. These latter focus mainly on solving a particular OPF problem (e.g., determination of system loadability limit) by handling the generator status switch between two possible states: either under voltage control or under field current limitation. The resulting MPEC problem is solved either directly [21] (e.g. by the LOQO commercial solver [11]), or by an iterative algorithm relying on the gradient projection method and aimed to identify binding constraints at the loadability limit [22]. Finally, in [23] a solution strategy is proposed that has some similarities with some MPEC approaches [16]. Its main feature is the handling of the perturbed complementarity constraints of the first order optimality conditions (obtained as in the IPA) by means of nonlinear complementarity functions [23].

In this paper two approaches to this problem are presented. The first one consists in reformulating the MINLP problem as a MPEC, which is solved by a modified IPA. The second approach includes in the OPF problem a nonlinear constraint approximating the integral constraint which limits the number of control actions allowed (this constraint has been previously used in the context of a fuzzy formulation of the OPF problem of generation cost minimization [7]). This problem has been solved by a successive linear programming (SLP) algorithm and hence this constraint has been used in a linearized form within SLP iterations. Some sophisticated heuristics have been pointed out to handle the effect of constraint linearization. In this work we use the same constraint but in the more general context of NLP nonlinear OPF model, solved by a modified IPA. Another contribution of the paper is the improvement of the integral constraint handling.

The paper is organized as follows. Section II briefly describes the classical OPF problem. Section III introduces the OPF problem with limited number of control actions. Two approaches to this problem are presented in Section IV. Section V provides some numerical results while Section VI concludes. The Appendix provides a brief description of the four main classes of approaches used to transform the MPEC into a NLP.

\section{Classical Optimal Power Flow problem}

The classical OPF problem can be written as follows:

$$
\begin{aligned}
& \min _{\mathbf{x}, \mathbf{u}} f(\mathbf{x}, \mathbf{u}) \\
& \text { s.t. } \\
& \mathbf{g}(\mathbf{x}, \mathbf{u})=\mathbf{0} \\
& \mathbf{h}(\mathbf{x}, \mathbf{u}) \leq \mathbf{0} \\
& \quad \underline{\mathbf{u}} \leq \mathbf{u} \leq \overline{\mathbf{u}}
\end{aligned}
$$

where $\mathbf{x}$ is the vector of state variables (i.e., real and imaginary part of voltage at all buses), $\mathbf{u}$ is the vector of control variables (e.g., generators active power, generators voltage (when controllable), Load Tap Changer (LTC) transformer ratios, shunt element reactances, load curtailment controls,

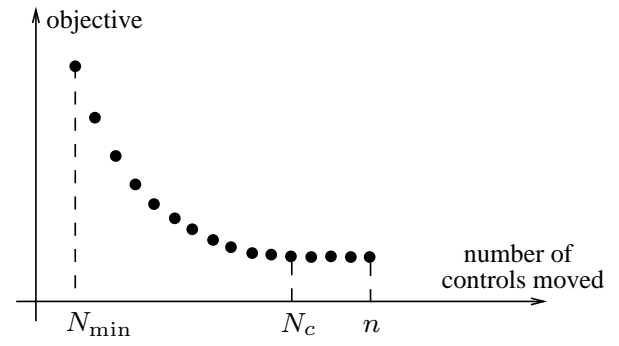

Fig. 1. Objective function versus number of controls allowed to move

phase shifters angle, etc.) and $\underline{\mathbf{u}}$ (resp. $\overline{\mathbf{u}}$ ) is its corresponding vector of lower (resp. upper) bounds, $f(\cdot)$ is the objective function, $\mathbf{g}(\cdot)$ and $\mathbf{h}(\cdot)$ are vectors of functions which model equality and inequality constraints. Equality constraints (2) are essentially the AC bus power equations, inequality constraints (3) refer to operational limits (e.g., branch currents and voltage magnitudes) while inequality constraints (4) refer to physical limits of equipments (e.g., bounds on: generators active/reactive powers, LTCs transformers ratio, shunts reactance, phase shifters angle, etc.).

\section{Optimal Power Flow With Limited Number of CONTROLS ALLOWED TO MOVE}

\section{A. Intuitive view of the problem}

In Figure 1, a plot of the objective function value versus numbers of controls allowed to move, in a general case is shown. In this figure $n$ is the total number of controls of the OPF problem. Clearly, two numbers of controls are particularly important when assessing the trade-off between the objective value and the number of control actions used in the OPF, namely (i) the minimal number of controls allowed to move such that to ensure OPFLC problem feasibility, which we denote by $N_{\min }$, and the minimal number of controls beyond which the objective can not be improved, i.e. the number of controls which has effectively moved in the classical OPF approach (1-4), which we denote by $N_{c}$. Thus, the number of controls $N$ for which the objective value varies satisfies: $0 \leq N_{\min } \leq N \leq N_{c} \leq n$.

\section{B. Problem statement}

Let us assume that an inital base case is available in terms of required OPF data ${ }^{1}$ (e.g., system topology, generator powers, loads powers, etc.), and let $\mathbf{u}^{0}=\left[u_{1}^{0}, \ldots, u_{i}^{0}, \ldots, u_{n}^{0}\right]^{T}$ be the vector of base case values of control variables. A stronger assumption is that the base case stems from a real-time operating point, or an off-line converged power flow solution.

\footnotetext{
${ }^{1}$ it is not mandatory that the system has a power flow solution
} 
The OPFLC problem can be compactly stated as follows:

$$
\begin{array}{rl}
\min _{\mathbf{x}, \mathbf{u}, \mathbf{s}} & f(\mathbf{x}, \mathbf{u}) \\
\text { s.t. } & \mathbf{g}(\mathbf{x}, \mathbf{u})=\mathbf{0} \\
& \mathbf{h}(\mathbf{x}, \mathbf{u}) \leq \mathbf{0} \\
& s_{i}\left(\underline{u}_{i}-u_{i}^{0}\right) \leq u_{i}-u_{i}^{0} \leq s_{i}\left(\bar{u}_{i}-u_{i}^{0}\right) \quad i=1, \ldots, n \\
& \sum_{i=1}^{n} s_{i} \leq N \\
& s_{i} \in\{0,1\} \quad i=1, \ldots, n
\end{array}
$$

where $\mathbf{u}=\left[u_{1}, \ldots, u_{i}, \ldots, u_{n}\right]^{T}$ is the vector of control variables, $\underline{\mathbf{u}}=\left[\underline{u}_{1}, \ldots, \underline{u}_{i}, \ldots, \underline{u}_{n}\right]^{T}, \overline{\mathbf{u}}=\left[\bar{u}_{1}, \ldots, \bar{u}_{i}, \ldots, \bar{u}_{n}\right]^{T}$, $\mathbf{s}=\left[s_{1}, \ldots, s_{i}, \ldots, s_{n}\right]^{T}$ is the vector of control variables status, a status equal to 1 (resp. 0) means that the control variable can (resp. can not) be moved, and $N$ is the specified maximum number of controls movements allowed.

Constraints (10) ensure that the status of any control variable is either 0 or 1 . Constraints (8) impose bounds on control variables movement with respect to the base case and are adapted from (4). Observe that if the status of a control variable is equal to 1 (resp. 0) the latter is allowed to vary between its bounds, (8) and (4) being equivalent (resp. is frozen to its base case value $u_{i}^{0}$ ). Finally, constraint (9) imposes an upper limit on the number of controls that can be moved and can be seen as policy constraint in power system operation.

The solution of the OPFLC problem is very useful under the following conditions: (i) the system does not have an equilibrium point (i.e., constraints (6) are not met), (ii) at the given operating point some operational limits are violated (i.e., constraints (7) are not satisfied), or (iii) the system operation state is feasible (i.e., constraints (6-7) are met) but non optimal, letting thus room to improve the objective function and thus to move to a more convenient operation state.

Observe that in the formulation (5-10), for simplicity, controls which may move in discrete steps (e.g., LTC tap changes, shunt compensation steps) have been modeled in the same way as continuous controls (e.g., generators active power, etc.). This means that moving several discrete steps is seen as a single control action. However, the model could be straightforwardly extended so as to take into account a discrete step movement as a single control action.

The OPFLC problem (5-10) constitutes, in its general form, a MINLP. However, solving the OPFLC problem as a MINLP is computationally expensive, e.g., an enumerative solution approach to this MINLP requires the solution of $\sum_{i=1}^{N} C_{n}^{i}=\frac{n !}{i !(n-i) !}$ classical OPF problems corresponding to all combinations of status variables values satisfying the constraint (9). For instance, even for a small size problem with $n=10$ and $N=5$ any MINLP approach has to choose among 637 integer combinations of status variables. To avoid such extensive computations the traditional approach to the OPFLC consists in solving the problem for beforehand specified values of control variables status $\mathbf{s}$, and most often by using $s_{i}=1$ $(i=1, \ldots, n)[1]-[4]$.
C. OPFLC: minimization of the number of controls allowed to move

An interesting particular case of the OPFLC problem is the minimization of the number of controls allowed to move [1], [2]. In this case the objective function (5) takes on the form:

$$
f(\mathbf{x}, \mathbf{u})=\sum_{i=1}^{n} s_{i}
$$

and $N_{\min }=\min \sum_{i=1}^{n} s_{i}$ is the optimal value which ensures OPFLC problem feasibility.

Note that, for this particular objective, the constraint (9) is removed from the formulation.

The determination of $N_{\text {min }}$ provides very valuable information, since it helps choosing $N$ in OPFLC formulation so as to ensure problem feasibility, e.g., by choosing $N \geq N_{\min }$.

\section{FIRST APPROACH: SOLVING OPFLC AS MPEC}

\section{A. Problem statement}

The original OPFLC problem (5-10) can be transformed into an equivalent MPEC problem, which we label OPFLC-MPEC:

$$
\begin{aligned}
& \min _{\mathbf{x}, \mathbf{u}, \mathbf{s}} f(\mathbf{x}, \mathbf{u}) \\
& \text { s.t. } \mathbf{g}(\mathbf{x}, \mathbf{u})=\mathbf{0} \\
& \mathbf{h}(\mathbf{x}, \mathbf{u}) \leq \mathbf{0} \\
& \underline{\mathbf{u}} \leq \mathbf{u} \leq \overline{\mathbf{u}} \\
& \left(1-s_{i}\right)\left(u_{i}-u_{i}^{0}\right)^{2}=0 \quad i=1, \ldots, n \\
& s_{i}\left(1-s_{i}\right)=0 \quad i=1, \ldots, n \\
& \sum_{i=1}^{n} s_{i} \leq N
\end{aligned}
$$

where constraint (17) replaces (10) by forcing controls status $s_{i}$ 's to take only values 0 or 1 . Observe also that constraints (8) and (10) of OPFLC formulation have been replaced by equivalent constraints $(15,16,17)$.

Note that the OPFLC-MPEC problem is highly non-convex, due to the large set of discrete equilibria (stemming from strict complementarity of solutions); hence, only local solutions can be guaranteed. The feasible region of the problem is the reunion of individual feasibility regions associated to all possible values of status variables $s_{i} \in\{0,1\}, \forall i=1, \ldots, n$ satisfying (18), and for which there exists continuous solution $(\mathbf{x}(\mathbf{s}), \mathbf{u}(\mathbf{s}))$ satisfying (13-15). Thus, the feasible region of OPFLC-MPEC problem may be composed of many disjoint regions.

\section{B. Solution strategy: relaxation approach}

We transform the OPFLC-MPEC problem (12-18) into a NLP problem by an approach based on the relaxation ${ }^{2}$ of complementarity constraints (16) and (17) [13]. We next solve the NLP problem by a modified IPA.

\footnotetext{
${ }^{2}$ The four main classes of approaches used to transform the MPEC into a NLP are briefly presented in the Appendix
} 
The OPFLC-MPEC relaxation approach takes on the form:

$$
\begin{array}{ll}
\min _{\mathbf{x}, \mathbf{u}, \mathbf{s}} & f(\mathbf{x}, \mathbf{u}) \\
\text { s.t. } & \mathbf{g}(\mathbf{x}, \mathbf{u})=\mathbf{0} \\
\mathbf{h}(\mathbf{x}, \mathbf{u}) \leq \mathbf{0} & \\
\underline{\mathbf{u}} \leq \mathbf{u} \leq \overline{\mathbf{u}} & \\
& \left(1-s_{i}\right)\left(u_{i}-u_{i}^{0}\right)^{2} \leq \theta \quad i=1, \ldots, n \\
& \\
\sum_{i=1}^{n} s_{i} \leq N & \\
0 \leq s_{i} \leq 1 & i=1, \ldots, n
\end{array}
$$

where complementarity constraints (17) have been relaxed as constraints (25) and complementarity constraints (16) are relaxed by a parameter $\theta>0$, so as to guarantee a strictly feasible interior for the inequality constraints. In this approach the relaxation parameter $\theta$ must be driven to 0 in the limit.

The barrier problem corresponding to (19-25) can be straightforwardly obtained as in IPM [24]-[27]:

$$
\begin{aligned}
& \min _{\mathbf{x}, \mathbf{u}, \mathbf{s}, \mathbf{z}} f(\mathbf{x}, \mathbf{u})-\mu \sum_{i=1}^{m} \ln z_{1 i} \\
& -\mu \sum_{i=1}^{n}\left(\ln z_{2 i}+\ln z_{3 i}+\ln z_{4 i}\right) \\
& -\mu \ln z_{5}-\mu \sum_{i=1}^{n}\left(\ln z_{6 i}+\ln z_{7 i}\right) \\
& \text { s.t. } \mathbf{g}(\mathbf{x}, \mathbf{u})=\mathbf{0} \\
& -\mathbf{h}(\mathbf{x}, \mathbf{u})-\mathbf{z}_{1}=\mathbf{0} \\
& \mathbf{u}-\underline{\mathbf{u}}-\mathbf{z}_{2}=\mathbf{0} \\
& \overline{\mathbf{u}}-\mathbf{u}-\mathbf{z}_{3}=\mathbf{0} \\
& \left(1-s_{i}\right)\left(u_{i}-u_{i}^{0}\right)^{2}+z_{4 i}=\theta \quad i=1, \ldots, n \\
& N-\sum_{i=1}^{n} s_{i}-z_{5}=0 \\
& s_{i}-z_{6 i}=0 \\
& 1-s_{i}-z_{7 i}=0
\end{aligned}
$$

where $\mu$ is the barrier parameter, $m$ is the dimension of vector

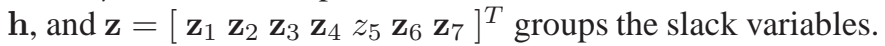

We solve the barrier problem (26-34) for a decreasing sequence of parameters $(\mu, \theta) \rightarrow 0$.

The perturbed Karush-Kuhn-Tucker (KKT) first order optimality conditions of the barrier problem (26-34) can be obtained likewise as in the IPM [24]-[27], while the exact conditions are obtained in the limit for $\mu=0$ and $\theta=0$.

\section{Algorithm of OPFLC-MPEC approach}

The outline of the algorithm used to solve the OPFLCMPEC problem is as follows:

1) Set $k \leftarrow 0$. Initialize variables as in IPA [24]-[27]. Choose relaxed and normal convergence tolerances $\varepsilon^{r}>$ 0 and $0<\varepsilon<\varepsilon^{r}$, respectively. Choose $\mu^{k}>0$ and $\theta^{k}>0$.

2) Perform an IPA iteration for the solution of the barrier problem (26-34) for the current value of $\mu^{k}$ and $\theta^{k}$.
Set $k \leftarrow k+1$.

Let $\mu_{I P A}^{k}$ be the estimate of the barrier parameter for the next iteration within the IPA.

3) Check approximate convergence. If either primal feasibility or dual feasibility [24]-[27] of the barrier problem (26-34) is larger than $\varepsilon^{r}$, go to step 2.

4) Check convergence. A (locally) optimal solution is found and the algorithm terminates when the following conditions are satisfied: primal feasibility and dual feasibility are smaller than $\varepsilon$, complementarity gap, objective function variation from an iteration to the next, the barrier parameter, and the relaxation parameter fall below some pre-defined tolerances [24]-[27].

5) Update the barrier parameter and the relaxation parameter:

$$
\begin{aligned}
\mu^{k} & \leftarrow \max \left\{\mu_{I P A}^{k}, \beta \mu^{k-1}, \mu_{\min }\right\} \\
\theta^{k} & \leftarrow \max \left\{\theta^{k-1} \frac{\mu^{k}}{\mu^{k-1}}, \theta_{\min }\right\}
\end{aligned}
$$

where $0<\beta<1$, and $\mu_{\min }$ and $\theta_{\min }$ are chosen equal to their respective convergence tolerance. Go to step 2 .

The features of this algorithm are:

- we use the multiple centrality corrections (MCC) IPA [24], [27] for the solution of barrier problems of step 2;

- each barrier problem is solved with the accuracy $\varepsilon^{r}$;

- slower decrease of the barrier parameter as compared to the MCC IPA;

- the relaxation parameter $\theta$ is updated in the same proportion as the barrier parameter $\mu$; both being driven to 0 in the limit.

\section{SECOND APPROACH: INTEGRAL CONSTRAINT APPROXIMATION}

\section{A. Problem statement}

An alternative formulation of the original OPFLC problem (5-10) is to approximate the integral constraint (9) by smooth nonlinear function (41), as in [7], which leads to the problem:

$$
\begin{aligned}
& \min _{\mathbf{x}, \mathbf{u}} f(\mathbf{x}, \mathbf{u}) \\
& \text { s.t. } \mathbf{g}(\mathbf{x}, \mathbf{u})=\mathbf{0} \\
& \mathbf{h}(\mathbf{x}, \mathbf{u}) \leq \mathbf{0} \\
& \underline{\mathbf{u}} \leq \mathbf{u} \leq \overline{\mathbf{u}} \\
& \quad \sum_{i=1}^{n} \frac{\left(u_{i}-u_{i}^{0}\right)^{2}}{\alpha_{i}+\left(u_{i}-u_{i}^{0}\right)^{2}} \leq N
\end{aligned}
$$

where $\alpha_{i}>0$ is a small positive number. Observe that the above approach differs from the classical OPF formulation (1-4) only by constraint (41). The latter approximates the true integral constraint (9), preventing thus the optimization problem from being a MINLP problem. Thus the status $s_{i}$ of control variable $u_{i}$ is approximated by $s_{i} \simeq \frac{\left(u_{i}-u_{i}^{0}\right)^{2}}{\alpha_{i}+\left(u_{i}-u_{i}^{0}\right)^{2}}$. Obviously, the smaller $\alpha_{i}$ the better the approximation of the true integral constraint; hence, in the limit $\left(\alpha_{i} \rightarrow 0\right)$, the status of variable $u_{i}$ either $s_{i} \rightarrow 0$ or $s_{i} \rightarrow 1$. Henceforth, this approach is called OPFLC-ICA, ICA standing for integral constraint approximation. 
TABLE I

TEST SYSTEMS SUMMARY

\begin{tabular}{c|c|c|c|c|c|c|c|c}
\hline system & $n$ & $g$ & $d$ & $b$ & $l$ & $t$ & $o$ & $s$ \\
\hline \hline Nordic32 & 60 & 23 & 22 & 81 & 57 & 31 & 4 & 12 \\
\hline IEEE118 & 118 & 54 & 91 & 186 & 175 & 11 & 9 & 14 \\
\hline
\end{tabular}

Note that constraint (41) has been used in the context of a fuzzy formulation of the OPF problem of generation cost minimization [7]. The latter problem has been solved by a SLP algorithm and hence this constraint has been used in a linearized form within SLP iterations. Some sophisticated heuristics have been proposed to handle the effect of this constraint linearization. Moreover, to prevent numerical problems, $\alpha_{i}$ 's have been kept constant during SLP iterations and equal to $\alpha_{i}=0.01\left(\bar{u}_{i}-\underline{u}_{i}\right)$.

In this work we use the constraint (41) in the context of full NLP OPF formulation and drive $\alpha_{i}$ 's to zero at the same pace with the barrier parameter $\mu$. The main difficulty of an IPA to solve (37-41) is that the feasible region shifts when $\alpha_{i}$ decreases, which can lead to algorithm stalling, especially for small values of $\alpha_{i}$ 's.

\section{B. Algorithm to solve OPFLC-ICA problem}

The algorithm to solve the OPFLC-ICA problem has the same steps as that for the solution of OPFLC-MPEC problem (see Section IV-C), except of:

- $\alpha_{i}$ 's took the role of $\theta$;

- $\alpha_{i}$ 's are initialized as: $\alpha_{i}^{0}=0.05\left(\bar{u}_{i}-\underline{u}_{i}\right)$;

- $\alpha_{i}$ 's are updated at setp 5 as:

$$
\alpha_{i}^{k} \leftarrow \alpha_{i}^{k-1} \frac{\mu^{k}}{\mu^{k-1}}
$$

- to prevent feasible region shifing a lower bound may be imposed for $\alpha_{i}$ 's, e.g., $\alpha_{i}=0.0001\left(\bar{u}_{i}-\underline{u}_{i}\right)$.

\section{NUMERICAL RESULTS}

\section{A. Test systems characteristics}

In this section we present some numerical results obtained with the proposed approaches on two test systems: a 60-bus system, which is a modified variant of the Nordic32 system [28], and the IEEE118 system [29].

A summary of their characteristics is given in Table I, where: $n, g, d, b, l, t, o, s$, and $c$ denote the number of: buses, generators, loads, branches, lines, transformers, transformers with controllable ratio, and shunt elements, respectively.

All tests have been performed on a PC 1.7-GHz Pentium IV with 512-Mb RAM.

\section{B. Removing thermal congestions at minimal load curtailment cost}

We focus on the OPF problem of minimum load curtailment cost to remove thermal congestions. We consider continuous load curtailment only (compensated by generators participating in frequency regulation) as control variable. Equality constraints are the $\mathrm{AC}$ bus active/reactive power flow equations
TABLE II

COMPARISON OF PROPOSED APPROACHES ON NORDIC32 SYSTEM

\begin{tabular}{c|c|c|c|c|c|c|c|c}
\hline \multirow{2}{*}{$N$} & \multicolumn{5}{|c|}{ MPEC } & \multicolumn{4}{c}{ ICA } \\
\cline { 2 - 9 } & $n_{e f}$ & obj & iter & time & $n_{\text {ef }}$ & obj & iter & time \\
\hline \hline 2 & & & & & 2 & 316.60 & 76 & 1.06 \\
\hline 3 & 3 & 303.94 & 82 & 1.62 & 2 & 316.60 & 58 & 0.83 \\
\hline 4 & & & & & 3 & 307.71 & 59 & 0.87 \\
\hline 5 & 5 & 289.97 & 89 & 1.70 & 5 & 281.36 & 93 & 1.41 \\
\hline 6 & 6 & 278.33 & 58 & 0.96 & 5 & 284.62 & 56 & 0.78 \\
\hline 7 & 6 & 273.40 & 54 & 0.93 & 6 & 278.32 & 81 & 1.16 \\
\hline 8 & 6 & 272.44 & 54 & 0.93 & 8 & 270.96 & 166 & 2.50 \\
\hline 9 & 6 & 272.44 & 54 & 0.93 & 8 & 270.96 & 56 & 0.80 \\
\hline 10 & 8 & 270.96 & 54 & 0.93 & 8 & 270.96 & 55 & 0.78 \\
\hline
\end{tabular}

and imposed voltages of generators. Inequality constraints are bounds on generator active/reactive powers, limits on branch currents and maximum allowed percentage of load curtailment at a bus, which we assume as $10 \%$ of the total bus load. Load curtailment is performed under constant power factor.

We solve this problem for the Nordic32 system only. We consider that following a line outage two branches are overloaded of $14 \%$ and $16 \%$, respectively. We also assume that these overloads have to be removed very quickly which prevents calling cheaper generation rescheduling but only to act on faster and more expensive load curtailment.

When using the classical OPF approach, 8 loads (i.e., $N_{c}=8$ ) share the effort of overloads removal, the MCC IPA converging in 10 iterations while the CPU times of optimization process being of 0.12 seconds.

Table II provides the results (number of controls effectively moved $n_{e f}$, objective value, number of iterations to convergence and CPU times of the optimization process, given in seconds) obtained with both OPFLC approaches (MPEC and ICA) for various values of maximal number of controls allowed to move $N$. In this case the minimal number of controls allowed to move so as to ensure problem feasibility is $N_{\min }=2$.

The settings of IPA to solve the MPEC and ICA approaches are given in Sections IV-C and V-B, respectively.

The settings used for the MPEC approach (see MPEC algorithm of Section IV-C) are: $\mu^{0}=1, \theta^{0}=1, \mu_{\min }=10^{-6}$, $\theta_{\min }=10^{-4}, \varepsilon=10^{-4}, \varepsilon^{r}=10^{-1}$, and $\beta=0.8$, respectively.

By looking closely to the results of this Table one can notice that:

1) the number of iterations to convergence is in average about 6 (resp. 8) times higher for MPEC (resp. ICA) approach with respect to the classical OPF;

2) the CPU times is in average about 10 times higher for both MPEC and ICA approaches compared to the classical OPF;

3) the ICA approach is more robust than the MPEC one, since the latter fails to converge for two values of $N$. These convergence problems can however be overcome by slightly shifting the IPA settings (e.g., different scheme of decreasing the barrier parameter, different initial conditions, etc.) or applying another IPA such as: predictor-corrector or pure primal-dual. Also according to our experience the ICA approach is more robust 
than MPEC with respect to reasonable changes of IPA settings;

4) sometimes the number of controls effectively moved is slightly lower than the maximum number allowed, $n_{e f}<N$. This happens in 2 cases (out of 7) for ICA approach;

5) sometimes in order to find solutions where the number of controls effectively moved is equal to the number of controls of classical OPF $\left(n_{e f}=N_{c}\right)$ one needs to impose $N$ slightly higher than $N_{c}$. This holds true especially for MPEC approach;

6) sometimes when incrementing $N$ it is possible that the OPFLC converges to the same solution (e.g., incrementing $N$ from 8 to 9 (resp. 2 to 3) for MPEC (resp. ICA) approach);

7) it is sometimes possible that an increment of $N$ leads to a worse objective (e.g., incrementing $N$ from 5 to 6 for ICA approach).

The items 4 to 7 mentioned above lead to suboptimal behaviour of MPEC and ICA approaches. Despite these light drawbacks, the ICA approach provides satisfactory results.

As regards the ICA approach, we have observed that, when $\alpha_{i}$ gets very small it sometimes happens that a very small movement of a control variable makes its status $s_{i}$ tending to 1 provided that $\alpha_{i}$ approaches 0 (much) faster than $\left(u_{i}-u_{i}^{0}\right)^{2}$. In this case the number of controls which have moved a reasonable amount is slightly lower than $N$, leading thus to sub-optimal solution. This situation has been also encountered in [7] and is the price to pay for using this constraint. A possibility to partially avoid such situation is to keep on decreasing $\alpha_{i}$ only for those variables $u_{i}$ for which a significant movement $\left(u_{i}-u_{i}^{0}\right)$ is observed and keep $\alpha_{i}$ constant for a variable as soon as the condition " $u_{i}-u_{i}^{0}$ gets small while $\frac{\left(u_{i}-u_{i}^{0}\right)^{2}}{\alpha_{i}+\left(u_{i}-u_{i}^{0}\right)^{2}}$ gets large" is detected.

\section{Restoring a system equilibrium point at minimum load curtailment cost}

We focus on the OPF problem of minimum load curtailment cost to restore a system equilibrium point. We consider again continuous load curtailment only as control variable. Equality constraints are the $\mathrm{AC}$ bus active/reactive power flow equations and imposed voltage of generators. Inequality constraints are bounds on generator active power, limits on branch currents and maximum allowed percentage of load curtailment at a bus, which we assume as $10 \%$ of the total bus load. Load curtailment is performed under constant power factor. Generators reactive power limits have not been considered so as to increase the degree of nonlinearity of the problem, or in other words to make sure that the optimum always corresponds to a saddle node bifurcation.

We solve this problem for IEEE118 system only. For the sake of testing the approaches under stringent conditions we have built a base case where the system load has been increased with $2 \%$ above the system loadability limit. This load increase has been performed proportionally to the initial consumption of each individual load. Clearly, for this new load
TABLE III

COMPARISON OF PROPOSED APPROACHES ON IEEE118 SYSTEM

\begin{tabular}{c|c|c|c|c|c|c|c|c}
\hline \multirow{2}{*}{$N$} & \multicolumn{4}{|c|}{ MPEC } & \multicolumn{4}{c}{ ICA } \\
\cline { 2 - 9 } & $n_{\text {ef }}$ & obj & iter & time & $n_{e f}$ & obj & iter & time \\
\hline \hline 8 & & & & & 8 & 721.0 & 72 & 1.84 \\
\hline 9 & & & & & & & & \\
\hline 10 & & & & & & & & \\
\hline 11 & & & & & 11 & 452.6 & 69 & 1.82 \\
\hline 12 & & & & & 11 & 469.9 & 75 & 1.94 \\
\hline 13 & & & & & 12 & 372.3 & 66 & 1.72 \\
\hline 14 & 12 & 456.9 & 117 & 5.06 & 14 & 322.2 & 90 & 2.30 \\
\hline 15 & 14 & 384.2 & 262 & 11.03 & 14 & 321.4 & 102 & 2.75 \\
\hline 16 & 14 & 338.4 & 231 & 15.32 & 14 & 320.5 & 72 & 2.00 \\
\hline 17 & 16 & 215.4 & 231 & 13.64 & 15 & 314.4 & 61 & 1.56 \\
\hline 18 & 15 & 314.5 & 55 & 2.64 & 17 & 298.5 & 64 & 1.62 \\
\hline 19 & 15 & 314.5 & 60 & 2.87 & 17 & 298.0 & 59 & 1.55 \\
\hline 20 & 17 & 300.7 & 72 & 3.29 & 19 & 294.4 & 63 & 1.61 \\
\hline 21 & 17 & 297.8 & 55 & 2.50 & 19 & 294.4 & 64 & 1.72 \\
\hline
\end{tabular}

level the system does not have an equilibrium point (i.e., any power flow computation will diverge).

When using the classical OPF approach 19 loads share the effort of restoring a system equilibrium point (i.e., $N_{c}=19$ ), the MCC IPA converging in 26 iterations while the CPU times of optimization process being of 0.62 seconds.

Table III provides the results obtained with both OPFLC approaches (MPEC and ICA), using the same format as Table II. In this case the minimal number of controls allowed to move so as to ensure problem feasibility is $N_{\min }=8$.

The settings of IPA to solve the MPEC and ICA approaches are the same as in the previous example (see Sections IV-C and $\mathrm{V}-\mathrm{B}$, respectively).

Note first that all remarks made for the previous example hold for this example as well.

We have noticed that for this very nonlinear problem the MPEC approach behaves poorly, diverging for any value of $\mathrm{N}$ in the interval [ 813 . Divergence in these cases typically happens in the form of dual variables tending to infinity when narrowing the bounds of the complementarity constraints' interval. Despite various heuristic strategies used (see item 3 of previous Section) only in a very small number of such cases convergence has been finally achieved. We believe that this pathological behaviour is attributable to the high degree of nonlinearity of the problem rather than to the increase in problem size. Note also that the IPA algorithm used to solve the MPEC approach does not contain special techniques to deal with stringent MPEC problems, e.g., regularization techniques, merit functions, etc. [13], [15]. It is therefore expected that the use of ad hoc (commercial or academic) MPEC solvers (e.g., LOQO, IPOPT, etc.) would significanlty improve the robustness of MPEC approach.

On the other hand, the ICA approach behaves again satisfactorily, only two divergent cases have been observed, which have been fixed by IPA parameters shifting. Again, we have observed that ICA is quite robust with respect to reasonable variation of IPA parameter settings. Finally, despite the higher degree of problem nonlinearity and problem size, the number of iterations to convergence with ICA approach is in average very close to that of the previous example, and only 2-3 times larger than classical OPF approach. 


\section{CONCLUSION}

This paper has presented a formulation of the OPF problem with a pre-specified number of controls allowed to move. This tool is essential for the SO, especially in real-time environments, providing the desired number of controls so as to achieve a specified operation goal. Besides, this approach yields a trade-off between the objective value and the number of control actions used.

We have proposed and compared two alternative approaches to the solution of the OPFLC problem, MPEC and ICA. We have found that, when solved by an IPA without special features to deal with degeneracy cases, the MPEC approach often experiences numerical problems, especially for highly nonlinear OPF problems. Clearly, to decide whether this approach is viable to solve OPFLC problems it will be necessary to improve the robustness of the NLP solver used so as to handle such degenerate problems more effectively. On the other hand, the ICA approach is found to provide satisfactory results and we believe that it may be successfully applied to other OPF problems.

Future work concerns the extension of these techniques to other (real-time) OPF problems such as: the removal of (voltage and thermal) congestions or reactive power dispatch. Another extension of the present work is the corrective security-constrained OPF [30] with limited number of controls that can be rescheduled in post-contingency states.

\section{ACKNOWLEDGMENTS}

This paper presents research results of the Belgian Network DYSCO, funded by the Interuniversity Attraction Poles Programme, initiated by the Belgian State, Science Policy Office. Florin Capitanescu and Louis Wehenkel acknowledge their funding by the FP7 EC project PEGASE. William Rosehart acknowledges the financial support of the Belgium National Fund of Scientific Research (FNRS) of which he obtained a visiting professor grant in 2008. The scientific responsibility rests with its authors.

\section{APPENDIX}

\section{A. MPEC problem statement}

Mathematical Programs with Equilibrium Constraints (MPEC) can be generally stated as follows:

$$
\begin{aligned}
& \min _{\mathbf{y}} f(\mathbf{y}) \\
& \text { s.t. } \mathbf{g}(\mathbf{y})=\mathbf{0} \\
& \mathbf{h}(\mathbf{y}) \leq \mathbf{0} \\
& c_{1 i}(\mathbf{y}) c_{2 i}(\mathbf{y})=0 \\
& i=1, \ldots, p \\
& c_{1 i}(\mathbf{y}) \geq 0, \quad c_{2 i}(\mathbf{y}) \geq 0 \\
& i=1, \ldots, p
\end{aligned}
$$

where (46) are complementarity constraints, which express that at least one of functions $c_{1 i}(\mathbf{y})$ or $c_{2 i}(\mathbf{y})$ must be 0 . Complementarity is called strict if only one functions is 0 and non-strict if both are 0 .

Recently, due to the high robustness and maturity reached by NLP solvers, there have been a large interest to reformulate MPEC problems as NLPs, and solve them with classical NLP solvers (e.g., SQP, IPM, active set identification, etc.) [9]-[15].

\section{B. Main MPEC solution approaches}

Four classes of approaches can be distinguished for the solution of the MPEC problem using NLP solvers, as explained hereafter.

1) Equivalent NLP reformulation approach: The simplest reformulation of the MPEC problem consists in expressing complementarity constraints by inequality constraints [9][11]:

$$
\begin{array}{ll}
\min _{\mathbf{y}} f(\mathbf{y}) & \\
\text { s.t.g }(\mathbf{y})=\mathbf{0} & \\
\mathbf{h}(\mathbf{y}) \leq \mathbf{0} & \\
\quad c_{1 i}(\mathbf{y}) \geq 0, \quad c_{2 i}(\mathbf{y}) \geq 0 & i=1, \ldots, p \\
\quad c_{1 i}(\mathbf{y}) c_{2 i}(\mathbf{y}) \leq 0 & i=1, \ldots, p
\end{array}
$$

Alternatively, the $p$ constraints (52) can be aggregated to a single one [9]:

$$
\sum_{i=1}^{p} c_{1 i}(\mathbf{y}) c_{2 i}(\mathbf{y}) \leq 0,
$$

or be expressed as equality constraints [11].

The main difficulty in solving the NLP (48-52) is related to the failure of standard constraint qualifications (CQs), especially the weaker Mangasarian-Fromovitz constraint qualification $^{3}$, and often the stronger linear independence constraint qualification $^{4}$ [9], [11]. If satisfaction of CQs fail then the set of Lagrange multipliers at the optimum is unbounded which can cause problems for traditional NLP solvers. Indeed, NLP algorithms require the satisfaction of CQs in order to ensure convergence.

Regularization techniques and other safeguards to deal with degeneracy are required for NLP solvers so as to cope with the difficulties posed by NLP formulation (48-52) [11], [14]. The (active-set) SQP algorithms have been found particularly suitable for solving the NLP (48-52).

In the context of IPM, to cope with these difficulties, two main classes of approaches have been proposed, based on the principle of constraint relaxation or penalty functions.

2) Relaxation approach: The relaxation approach [11]-[13] consists in relaxing (52) as:

$$
c_{1 i}(\mathbf{y}) c_{2 i}(\mathbf{y}) \leq \theta_{i} \quad i=1, \ldots, p
$$

where the relaxation parameter $\theta_{i}>0$ is driven to 0 at the optimum together with the barrier parameter $\mu$ [11]-[13]. The IPM solver used should carefully manage the two conflicting goals: that of decreasing $\mu$, and consequently allowing approaching the feasible region boundary and that of restricting the interior of the feasible region by relaxing $\theta_{i}$ 's.

In adition, bounds on complementarity functions can be also relaxed, with a parameter $\delta_{i}>0[12]$ :

$$
c_{1 i}(\mathbf{y}) \geq-\delta_{i}, \quad c_{2 i}(\mathbf{y}) \geq-\delta_{i} \quad i=1, \ldots, p
$$

\footnotetext{
${ }^{3}$ i.e., there does not exist a single feasible point satisfying strict feasibility (when all inequality constraints are strict)

${ }^{4}$ i.e., the gradients of the active inequality constraints and the gradients of the equality constraints are linearly independent at the optimum
} 
Relaxing both (51) and (52) ensures a strict feasible domain. In the limit either $\theta_{i}$ or $\delta_{i}$ must be driven to 0 . The drawback of this approach concerns the heuristic strategy to decide which parameter must be driven to 0 for each complementarity constraint.

3) Penalization approach: This approach consists in appending complementarity constraints (46) to the objective as penalty functions [14]:

$$
\min _{\mathbf{y}} f(\mathbf{y})+\rho \sum_{i=1}^{p} c_{1 i}(\mathbf{y}) c_{2 i}(\mathbf{y})
$$

where $\rho>0$ is a penalty parameter. The critical point in penalty approaches is the finding of a good initial value of $\rho>0$ as well as a robust heuristic technique to (possibly) update during IPM iterations.

Note that a hybrid approach of both complementarity constraints relaxation and penalty functions has been also proposed [15]. Thus, (52) are relaxed as (53) and appended to the objective:

$$
\min _{\mathbf{y}} f(\mathbf{y})+\rho^{\prime} \sum_{i=1}^{p} \theta_{i}
$$

$\theta_{i}$ 's becoming decision variables.

4) Smoothing approach: Finally, the class of smoothing approaches [16] replaces complementarity constraints (46) by the smoothed min-function:

$$
\begin{aligned}
\phi_{\mu}\left(c_{1 i}(\mathbf{y}), c_{2 i}(\mathbf{y})\right)= & \sqrt{\left(c_{1 i}(\mathbf{y})-c_{2 i}(\mathbf{y})\right)^{2}+4 \mu^{2}} \\
& -c_{1 i}(\mathbf{y})-c_{2 i}(\mathbf{y})=0, \quad i=1, \ldots, p
\end{aligned}
$$

which has the property:

$$
\begin{array}{r}
\phi_{\mu}\left(c_{1 i}(\mathbf{y}), c_{2 i}(\mathbf{y})\right)=0 \leftrightarrow \\
c_{1 i}(\mathbf{y}) \geq 0, c_{2 i}(\mathbf{y}) \geq 0, c_{1 i}(\mathbf{y}) c_{2 i}(\mathbf{y})=\mu^{2}
\end{array}
$$

and

$$
\phi_{0}\left(c_{1 i}(\mathbf{y}), c_{2 i}(\mathbf{y})\right)=-2 \min \left(c_{1 i}(\mathbf{y}), c_{2 i}(\mathbf{y})\right) .
$$

This smoothed problem is solved for decreasing positive values of parameter $\mu \rightarrow 0$ by a non-interior point algorithm.

\section{REFERENCES}

[1] B. Stott and E. Hobson,"Power system security control calculations using linear programming, Parts I and II", IEEE Trans. PAS, vol. PAS97, 1978, pp. 1713-1731.

[2] B. Stott, O. Alsac, and A.J. Monticelli, "Security analysis and optimization" (Invited Paper), IEEE Proc., vol. 75, no. 12, 1987, pp. 1623-1644.

[3] W.F. Tinney, J.M. Bright, K.D. Demaree, B.A. Hughes, "Some deficiencies in Optimal Power Flow", IEEE Trans. Power Syst. Vol. 3, No. 2, 1988, pp. 676-683.

[4] R. Bacher (Editors: K. Frauendorfer, H. Glavitsch, and R. Bacher), "Power system models, objectives and constraints in optimal power flow calculations" (chapter of the book "Optimization in Planning and Operation of Electric Power Systems"), Physica Verlag (Springer), Heidelberg, Germany, 1993, pp. 217-264.

[5] J.A. Momoh, R.J. Koessler, M.S. Bond, B. Stott, D. Sun, A. Papalexopoulos, and P. Ristanovic, "Challenges to optimal power flow", IEEE Trans. Power Syst. Vol. 12, No. 1, 1997, pp. 444-455.

[6] S.A. Soman, K. Parthasarathy, and D. Thukaram, "Curtailed number and reduced controller movement optimization algorithms for real time voltage/reactive power control", IEEE Trans. Power Syst., vol. 9, no. 4, November 1994, pp. 2035-2041.
[7] W.-H. Edwin Liu, and X. Gupa, "Fuzzy constraint enforcement and control action curtailement in an optimal power flow", IEEE Trans. Power Syst., vol. 11, no. 2, 1996, pp. 639-645.

[8] B.T. Baumrucker, J.G. Renfro, and L.T. Biegler, "MPEC problem formulations in chemical engineering applications", available online at http://dynopt.cheme.cmu.edu/papers/preprint/rp.pdf, 2007.

[9] M. Anitescu, "On solving mathematical programs with complementarity constraints as non-linear programs", Preprint ANL/MCS-P864-1200, Argonne National Laboratory, USA, 2000.

[10] R. Fletcher, S. Leyffer, D. Ralph, and S. Scholtes, "Local convergence of SQP methods For Mathematical Programming Problems with Equilibrium Constraints", Dundee Numerical Analysis Report NA/209, 2002.

[11] H.Y. Benson, D. Shanno, and R.J. Venderbei, "Interior-point methods for nonconvex nonlinear programming: complementarity constraints", Technical report ORFE-02-02, Operations Research and Financial Engineering, Princeton University, 2002.

[12] A.V. DeMiguel, M. P. Friedlander, F.J. Nogales, and S. Scholtes, "An interior-point method for MPECs based on strictly feasible relaxations", Technical report, London Business Shcool, IK, 2004.

[13] A.U. Raghunathan, and L.T. Biegler, "An interior-point method for mathematical programs with complementarity constraints (MPCCs)", SIAM Journal of Optimization, vol. 15, no. 3, pp. 720-750, 2005.

[14] S. Leyffer, G. Lopez-Calva, and J. Nocedal, "Interior methods for mathematical programs with complementarity constraints", Technical report OTC 2004-10, Optimization Technology Center, Northwestern University, USA, 2005.

[15] H.Y. Benson, A. Sen, D. Shanno, and R.J. Venderbei, "Interior-point algorithms, penalty methods and equilibrium problems", Technical report ORFE-03-02, Operations Research and Financial Engineering, Princeton University, 2003.

[16] F. Facchinei, H. Jiang, and L. Qi, "A Smoothing Method for Mathematical Programs with Equilibrium Constraints", Mathematical Programming, Vol. 85, 1999, pp. 107-134.

[17] B.F. Hobbs, C.B. Metzler, and J.S. Pang, "Strategic gaming analysis for electric power systems: an MPEC approach", IEEE Trans. Power Syst., vol. 15 , no. 2,2000 , pp. 638-645.

[18] M.L. Latorre and S. Granville, "The Stackelberg equilibrium applied to AC power systems - a non-interior point algorithm", IEEE Trans. Power Syst. vol. 18, no. 2, 2003, pp. 611-618.

[19] A.L. Motto and F.D. Galiana, "Coordination in markets with nonconvexities as a mathematical program with equilibrium constraints - Part I and II", IEEE Trans. Power Syst., vol. 19, no. 1, 2004, pp. 309-324.

[20] G. Bautista, M.F. Anjos, and A. Vannelli, "Formulation of oligopolistic competition in AC power networks: an NLP approach", IEEE Trans. Power Syst., vol. 22, no. 1, 2007, pp. 105-115.

[21] W. Rosehart, C. Roman, and A. Schellenberg, "Optimal power flow with complementarity constraints", IEEE Trans. Power Syst., vol. 20, no. 2, 2005, pp. 813-822.

[22] M.E. Karystianos, N.G. Maratos, and C.D. Vournas, "Maximizing Power-System Loadability in the Presence of Multiple Binding Complementarity Constraints", IEEE Trans. on Circuits and Systems I: Fundamental Theory and Applications, Vol. 54, No. 8, 2007, pp. 1775 - 1787.

[23] G.L. Torres, and V.H. Quintana, "Optimal Power Flow by a Nonlinear Complementarity Method", IEEE Transactions on Power Systems, Vol. 15 , No. 3, 2000, pp. 1028-1033.

[24] G.L. Torres, and V.H. Quintana, "On a Nonlinear Multiple-CentralityCorrections Interior-Point Method for Optimal Power Flow", IEEE Transactions on Power Systems, Vol. 16, No. 2, 2001, pp. 222-228.

[25] Y.C. Wu, A.S. Debs, and R.E. Marsten, "A Direct Nonlinear PredictorCorrector Primal-Dual Interior Point Algorithm for Optimal Power Flows", IEEE Trans. on Power Systems, Vol. 9, No. 2, 1994, pp. 876883.

[26] S. Granville, "Optimal reactive dispatch through interior point methods", IEEE Trans. on Power Systems, Vol. 9, No. 1, 1994, pp. 136-146.

[27] F. Capitanescu, M. Glavic, D. Ernst, and L. Wehenkel, "Interior-point based algorithms for the solution of optimal power flow problems", Electric Power Systems Research, vol. 77, no. 5-6, April 2007, pp. 508517.

[28] CIGRE Task Force 38.02.08, "Long-Term Dynamics, Phase II", 1995.

[29] IEEE-118 bus system, available online at http://www.ee.washington.edu, 1996.

[30] A.J. Monticelli, M.V.P. Pereira, and S. Granville, "Security-constrained optimal power flow with post-contingency corrective rescheduling", IEEE Trans. Power Syst., vol. PWRS-2, no. 1, February 1987, pp. 175182. 